\title{
Correction to: Blade Products and Angles Between Subspaces
}

\author{
André L. G. Mandolesi*®
}

\section{Correction to: Adv. Appl. Clifford Algebras (2021) 31:69 https://doi.org/10.1007/s00006-021-01169-w}

Unfortunately, the symbol $\mathcal{L}$, for partial orthogonality, has been incorrectly replaced by $\perp$. The instances in which one should read $\downarrow$ instead of $\perp$ are: page 5, Definition 2.2, "... (we write $V \downarrow W$ )..."

page 5, Proposition 2.3 (i), " $V \downarrow W \Leftrightarrow \ldots$.

page 5, Proposition 2.3 (ii), " $V \downarrow W \Leftrightarrow \ldots$.

page 5, Proposition 2.3 (iii), ". . then $V \downarrow W \Leftrightarrow W \downarrow V$."

page 5, second line before Definition 2.4, "Note that $\mathcal{L}$ is not..."

page 5, Definition 2.4 (i), "If $[A] \perp[B]$ we say ... and write $A \sim B$ ". Note that the $\perp$ in Definition 2.4 (ii) is correct.

page 6, second line of Proposition 2.7, " $A \downarrow B \Leftrightarrow A * B=0$."

page 7 , second line, " $\Theta_{V, W}=\frac{\pi}{2} \Leftrightarrow V \downarrow W$."

page 8, second line before Proposition 2.13, "implies $V \downarrow W^{\perp}$."

page 16, fourth line before Proposition 3.26, "and $V \not \chi W,(2)$ gives ..."

page 16, second line before Proposition 3.26, " $V \downarrow W$, both ..."

page 16, third line after Definition 3.27, "... and if $V \downarrow W$ we can ..."

page 24, second line of Corollary 5.3, " $A\rfloor B=0 \Leftrightarrow A \downarrow B$."

page 29, Corollary A.8, " $A\lrcorner B=0 \Leftrightarrow A \perp B$."

Also, reference [29] is missing some information. It should be:

[29] Mandolesi, A.L.G.: Projection factors and generalized real and complex Pythagorean theorems. Adv. Appl. Clifford Al. 30, 43 (2020). https://doi. org/10.1007/s00006-020-01070-y

Publisher's Note Springer Nature remains neutral with regard to jurisdictional claims in published maps and institutional affiliations.

The original article can be found online at https://doi.org/10.1007/s00006-021-01169-w.

*Corresponding author. 
André L. G. Mandolesi

Instituto de Matemática e Estatística

Universidade Federal da Bahia

Av. Adhemar de Barros s/n

Salvador BA40170-110

Brazil

e-mail: andre.mandolesi@ufba.br 